\title{
Unavoidable food waste estimate using food consumption data
}

\author{
Rita FS Sousa and Duarte PM Torres \\ Faculty of Nutrition and Food Sciences of the University of Porto Rua Dr. Roberto Frias, 4200-465 Porto \\ PORTUGAL
}

Food wasted through the food supply chain is getting to our attention, first because it raises ethical and economical concerns and second because wasted food can be used as raw material in different processes (ex. energy production or animal feed production). Total food waste can be classified as unavoidable or avoidable. The unavoidable part often corresponds to the inedible parts usually rejected during the preparation and consumption of foods. The aim of this study is to develop an approach to estimate the amount and composition of unavoidable waste using food consumption data.

Daily food consumption data of 86 food groups was obtained from a food frequency questionnaire (FFQ) applied to 417 adults ${ }^{(1)}$. The unavoidable food waste for each food group was calculated as the weighted average of the inedible fraction of related food items using data from food composition tables ${ }^{(2,3)}$ (inedible fraction) and the national household budget survey ${ }^{(4)}$ (weights of each food item in the related food group).

According to our study, on average, $271 \mathrm{~g}$ of unavoidable food waste/capita/day is produced: $173 \mathrm{~g}(64 \%)$ derive from inedible parts of fruit and vegetable products; $61 \mathrm{~g}(22 \%)$ come from inedible parts of meat, fish and dairy products; inedible parts of starchy roots and tubers (mainly potatoes) contributes with $11 \mathrm{~g}(4 \%)$; and the remaining $26 \mathrm{~g}(10 \%)$ derive from inedible parts of miscellaneous ingredients of common recipes like soups and pizzas.

Total unavoidable food waste annually produced in Portugal was roughly estimated as 998 kilotonne. This was done multiplying 271 (g of unavoidable food waste/capita/day) per 356 (day/year) per 10.1 million (Portuguese population above 5 years). The amount of inedible food waste annually produced in Portugal can be compared to the total amount of avoidable food waste estimated by other authors $^{(5)}$. Regarding food waste, in Portugal, the unavoidable/avoidable ratio is around 1 . In this study, two main methodological difficulties were only partially overtaken: first, the lack of robust data regarding the variability of inedible fraction of foods which depends mainly on food preparation habits; on the variety of cuts for fish and meat; and on the maturity state for fruits and vegetables. Second, the lack of food descriptors dedicated to food waste estimate that can be defined and captured during food intake assessment studies.

1. Lopes C, et al. (2006) Consumo Alimentar no Porto. Serviço de Higiene e Epidemiologia FMUP.

2. Martins I, et al. (2006) Food Composition Table. Instituto Nacional de Saúde Dr. Ricardo Jorge.

3. McCance Widdowson's (2002) The Composition of Foods. Sixth summary edition ed.: Cambridge, Royal Society of Chemistry.

4. INE (2005) National Household Budget Survey. Instituto Nacional de Estatística.

5. Baptista P, et al. (2012) Do Campo ao Garfo. Food Waste in Portugal, Lisboa: CESTRAS. 